\title{
Dietary Medium- and Long-chain Triacylglycerols Accelerate Diet-Induced Thermogenesis in Humans
}

\author{
Akiko Ogawa ${ }^{1}$, Naohisa Nosaka ${ }^{1}$, Michio Kasai ${ }^{1 *}$, Toshiaki Aoyama ${ }^{1}$, \\ Mitsuko Okazaki ${ }^{2}$, Osamu Igarashi ${ }^{3}$ and Kazuo Kondo ${ }^{4}$ \\ ${ }^{1}$ Health and Nutrition Science Division, Research Laboratory, The Nisshin OilliO Group, LTD. (1 Shinmei-cho, Yokosuka, Kanagawa, 239- \\ 0832, JAPAN) \\ ${ }^{2}$ Kagawa Nutrition University (3-9-21 Chiyoda, Sakado, Saitama 350-0288, JAPAN) \\ ${ }^{3}$ Ibaraki Christian University (6-11-1 Oomika-cho, Hitachi, Ibaraki, 319-1295, JAPAN) \\ ${ }^{4}$ Institute of Environmental Science for Human Life, Ochanomizu University (2-1-1 Otsuka, Bunkyo-ku, Tokyo 112-8610, JAPAN)
}

\begin{abstract}
This study investigated the effects of a liquid meal containing medium- and long-chain triacylglycerols (MLCT) on diet-induced thermogenesis (DIT) and was conducted in double-blind crossover manner. Twenty subjects participated in this examination. The subjects consumed the liquid meal, which was made with 14g of canola oil (LCT, long-chain triacylglycerols) or MLCT containing about 12\% medium-chain fatty acids (MCFA). Oxygen consumption and carbon dioxide production were measured by indirect calorimetry. Resting energy expenditure (REE) was determined based on there parameters, applying the equation of Weir. Increase in DIT after ingesting the liquid meal with MLCT during 6h was significantly greater than with LCT $(P<0.05)$. The results suggest that the substitution of MLCT for cooking oil is useful to control body weigh and fat in subjects.
\end{abstract}

Key words: medium- and long-chain triacylglycerol, diet-induced thermogenesis, human, cooking oil

\section{INTRODUCTION}

Obesity is a state in which excessive fat accumulates in the body due to energy intake exceeding energy expenditure. In particular, visceral fat accumulation is known to be one of the major risk factors for lifestyle-related diseases ${ }^{1,2)}$. Any individual with visceral fat accumulation associated with at least two of the three factors hyperlipidemia, hypertension and hyperglycemia is known to have a synergistically increased risk of developing arteriosclerosisrelated events such as myocardial infarction and cerebral infarction. It has recently been reported at the general meeting of the Japanese Society of Internal Medicine that this condition should be diagnosed as metabolic syndrome (multiple risk factor syndrome) $)^{3)}$. Currently in Japan, 50\% of men and 20\% of women aged 40-74 are reported to be at risk of developing metabolic syndrome, and prompt measures need to be taken for its prevention and treatment ${ }^{4)}$.

Diet-induced thermogenesis (DIT) accounts for around
$10 \%$ of daily energy expenditure and plays an important role in adjusting energy balance ${ }^{5}$. There have been numerous reports showing that medium-chain triacylglycerols (MCT) composed of 8-10 carbon atoms accelerate DIT $^{6-10)}$. Therefore, interest is currently focused on MCT as a lipid for preventing obesity. However, MCT has some problems in terms of its unsuitability as a cooking oil because of its low smoke point and foam generation when mixed with other vegetable oils and used for frying ${ }^{11}$. We therefore developed a new cooking oil containing triacylglycerols composed of medium- and long-chain triacylglycerols (MLCT), which are randomly interesterified triacylglycerols containing medium-chain fatty acids (MCFA) and long-chain fatty acids (LCFA) in the same glycerol molecule ${ }^{12)}$. MLCT has improved the cooking problems inherent to MCT, and can be used as a normal cooking oil. The present study was therefore conducted to determine whether intake of MLCT accelerates DIT, as is the case for MCT, in subjects.

\footnotetext{
* Correspondence to: Michio Kasai, Health and Nutrition Science Division Research Laboratory, The Nisshin OilliO Group, LTD., 1 Shinmeicho, Yokosuka, Kanagawa, 239-0832, JAPAN

E-mail: m-kasai@nisshin-oillio.com
}

Accepted February 21, 2007 (received for review January 29, 2007)

Journal of Oleo Science ISSN 1345-8957 print / ISSN 1347-3352 online

http://jos.jstage.jst.go.jp/en/ 


\section{EXPERIMENTAL}

\subsection{Subjects}

Twenty-one adults were enrolled in this study. One subject opted out of the study for personal reasons. Total subjects (9 males and 11 females: mean age $24.0 \pm 0.9$ years, height $164.7 \pm 2.3 \mathrm{~cm}$, weight $59.0 \pm 1.8 \mathrm{~kg}$, BMI $21.7 \pm$ $0.3 \mathrm{~kg} / \mathrm{m}^{2}$ ) were 20 adults. All the subjects had no history of hypertension, diabetes or hyperlipidemia. This study was approved by the Ethics Committee of Ochanomizu University and was carried out in conformity with the Declaration of Helsinki (established in 1964 and revised in 2000). All subjects gave informed consent to participate in the study after its objectives and methods had been fully explained.

\subsection{Test diet}

A liquid meal containing $14 \mathrm{~g}$ of MLCT (MLCT diet) was used. After the liquid meal had been prepared by mixing MLCT (14 g), casein (18.52 g), dextrin (75 g), and hot water (292.48 g) by vacuum emulsification, retort pouches were filled with the mixture, and retort sterilization was performed at $121.1^{\circ} \mathrm{C}$ and $2.5 \mathrm{~atm}$.

The liquid meal was stored at $5{ }^{\circ} \mathrm{C}$ and provided after reheating it to room temperature on the day before the study. As a control diet (food with long-chain triacylglycerols: LCT diet), a liquid meal containing canola oil instead of MLCT was used (Tables 1 and 2).

Table 1 Fatty Acid Composition of Test Oils.

\begin{tabular}{ccc}
\hline Fatty acid & $\begin{array}{c}\text { Long-chain }^{1} \\
\text { triacylglycerols }\end{array}$ & $\begin{array}{c}\text { Medium- and long-chain } \\
\text { triacylglycerols }\end{array}$ \\
\hline g:100g total fatty acids \\
$10: 0$ & $\mathrm{ND}^{3}$ & 8.7 \\
$16: 0$ & $\mathrm{ND}$ & 2.8 \\
$16: 1$ & 4.2 & 3.7 \\
$18: 0$ & 0.2 & 0.2 \\
$18: 1$ & 1.8 & 1.7 \\
$18: 2$ & 59.0 & 52.7 \\
$18: 3$ & 20.9 & 18.2 \\
$20: 0$ & 10.8 & 9.5 \\
$20: 1$ & 0.6 & 0.6 \\
$22: 0$ & 1.5 & 1.2 \\
$22: 1$ & 0.4 & 0.3 \\
$24: 0$ & 0.2 & $\mathrm{ND}$ \\
$24: 1$ & 0.2 & 0.2 \\
\hline
\end{tabular}

${ }^{1}$ Canola oil.

${ }^{2}$ Number of carbon atoms: number of double bonds.

${ }^{3} \mathrm{ND}$, not detected.

\subsection{Methods}

The study was conducted in a double-blind crossover manner. The subjects were instructed to maintain their usual lifestyle and diet during the study period. They assembled in a measuring room at 18:00 on the day before the study, and all of the subjects were given the same supper at 19:00. They were allowed to drink only water after supper. The measuring room was furnished with television sets, showers, beds, etc., and the subjects were allowed to spend their time freely within the room until bedtime at 23:00. The subjects woke up at 06:00 on the day of the study. Their height and weight were measured, and they lay relaxed in bed until measurement of expiratory gas at 06:30 before food intake. The subjects were given either the LCT diet or the MLCT diet, and postprandial expiratory gas was measured $30 \mathrm{~min}$ and $1 \mathrm{~h}$ later, and thereafter at 1-h intervals until $6 \mathrm{~h}$ after food intake. An AMIS 2000 SP (Innovision A/S, Denmark) was used for measurement of expiratory gas oxygen consumption and carbon dioxide production, and the values were used to calculate the nonprotein respiratory quotient $(R Q)$, and also resting energy expenditure (REE) using Weir's formula ${ }^{13)}$. Gas analysis was performed using a quadrupole mass spectrometer. After an interval of 2 days or more after the initial intake study, another study was performed with different oil. Temperature and humidity of the measuring room were set at 25$26^{\circ} \mathrm{C}$ and $50-55 \%$, respectively.

\subsection{Statistical analysis}

All values were expressed as the mean \pm standard error. Repeated two-way analysis of variance (oil x time) was performed for oxygen consumption and $\mathrm{RQ}$ after intake of the LCT diet and MLCT diet, and when significant differences were confirmed, paired t-test was performed for each time period. DIT was calculated using the trapezoidal method. One-way analysis of variance was performed for DIT after intake of the LCT diet and MLCT diet, and when significant differences were confirmed, paired t-test was used for analysis. In either case, a p value of less than $5 \%(\mathrm{p}<0.05)$ was considered to indicate statistical significance. SPSS Version 10.0J was used for statistical calculation.

Table 2 Nutrient and Energy Contents of the Liquid Meal $^{1}$.

\begin{tabular}{ll}
\hline Liquid meal & \\
\hline Energy (kcal) & 500 \\
Protein (\%) & 14.8 \\
Fat (\%) & 25.2 \\
Carbohydrate (\%) & 60.0 \\
\hline
\end{tabular}

${ }^{1}$ Liquid meal containing $14 \mathrm{~g}$ of long-chain triacylglycerols (LCT) or medium- and long-chain triacylglycerols (MLCT). 


\section{RESULTS}

REE before intake of the liquid meal, oxygen consumption and RQ in the 20 subjects were $1316.7 \pm 64.7 \mathrm{kcal}$, $186.4 \pm 9.0 \mathrm{~mL} / \mathrm{min}$, and $0.91 \pm 0.02$, respectively, in the LCT diet group, and $1312.0 \pm 62.6 \mathrm{kcal}, 184.6 \pm 8.7$ $\mathrm{mL} / \mathrm{min}$, and $0.94 \pm 0.02$, respectively, in the MLCT diet group.

Changes in oxygen consumption and RQ after intake of the liquid meal were similar in the LCT diet and MLCT diet groups (Fig. 1 and 2). Energy expenditure of DIT 6 hours after intake of the liquid meal was increased by approximately $14 \mathrm{kcal}$ in the MLCT diet group in comparison with the LCT diet group, indicating a $2.7 \%$ increase of energy expenditure in proportion to energy intake (Table 3).

\section{DISCUSSION}

In this study, we investigated how the intake of MLCT containing approximately 12\% MCFA influences DIT. The results showed that intake of MLCT significantly increased DIT. This is consistent with the results of Matsuo et al. showing that intake of MLCT containing 20\% MCFA significantly increased DIT ${ }^{11}$. Many studies have demonstrated that MCT increases DIT in comparison with LCT. Suzuki et al. conducted a study involving 25 volunteers who were given margarine containing $5 \mathrm{~g}$ of either LCT or MCT and reported that DIT increased by approximately $7 \mathrm{kcal}$ in the group given MCT as compared with that given $\mathrm{LCT}^{6)}$. Furthermore, Nosaka et al. reported that the amount of body fat, subcutaneous fat area, and visceral fat area were significantly reduced in the MCT group as compared with in the LCT group when 73 volunteers were given margarine containing $5 \mathrm{~g}$ of either LCT or MCT for 12 weeks under diet control ${ }^{14}$. This suggests that MCTinduced acceleration of DIT is one of the possible mechanisms by which MCT suppresses body fat accumulation. It has also been reported that MCT intake significantly

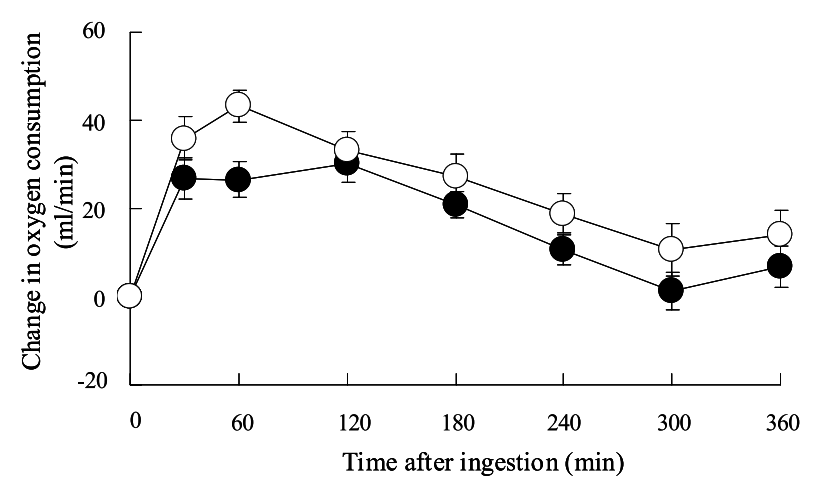

Fig. 1 Change in Oxygen Consumption before and after Ingestion of the Liquid Meal Containing $14 \mathrm{~g}$ of Long-chain Triacylglycerols or Medium- and Long-chain Triacylglycerols ${ }^{1}$.

${ }^{1}$ Mean $\pm \mathrm{SE}, \mathrm{n}=20$. ; long-chain triacylglycerols, $\bigcirc$; medium- and long-chain triacylglycerols.

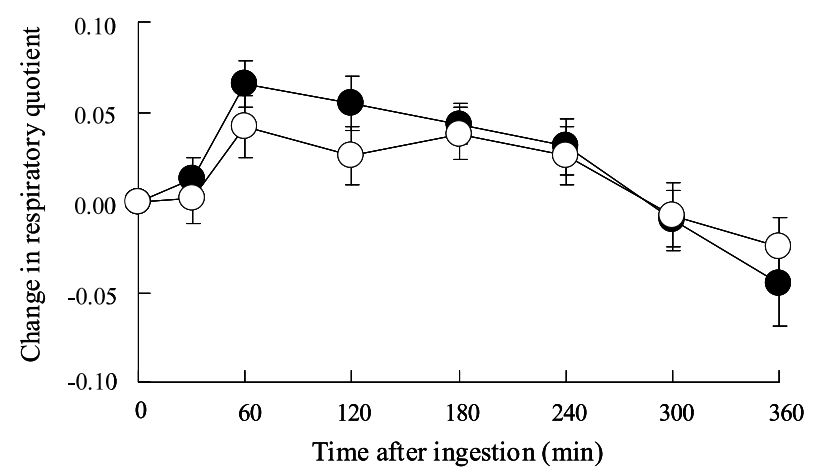

Fig. 2 Change in Respiratory Quotient before and after Ingestion of the Liquid Meal Containing $14 \mathrm{~g}$ of Long-chain Triacylglycerols or Medium- and Long-chain Triacylglycerols ${ }^{1}$.

${ }^{1}$ Mean $\pm \mathrm{SE}, \mathrm{n}=20$. ; long-chain triacylglycerols, $\bigcirc$; medium- and long-chain triacylglycerols.

Table 3 Diet-induced Thermogenesis During $6 \mathrm{~h}$ after Ingestion of the Liquid Meal Containing LCT or MLCT ${ }^{1}$.

\begin{tabular}{lrr}
\hline & \multicolumn{2}{c}{ Test meal $^{2}$} \\
\cline { 2 - 3 } & \multicolumn{1}{c}{ LCT } & \multicolumn{1}{c}{ MLCT } \\
\hline Diet-induced thermogenesis $\quad(\mathrm{kcal})$ & $34.4 \pm 3.6$ & $47.9 \pm 4.6^{*}$ \\
Rate of diet-induced thermogenesis $(\%)^{3}$ & $6.9 \pm 0.7$ & $9.6 \pm 0.9^{*}$ \\
\hline
\end{tabular}

${ }^{1}$ Mean $\pm \mathrm{SE}, n=20$.

${ }^{2}$ The liquid meal containing $14 \mathrm{~g}$ of long-chain triacylglycerols (LCT) or medium- and long-chain triacylglycerols (MLCT).

${ }^{3}$ Rate of diet-induced thermogenesis was calculated as diet-induced thermogenesis / energy of food consumption.

${ }^{*}$ Significantly different from the LCT diet, $p<0.05$. 


\section{A. Ogawa, N. Nosaka, M. Kasai et al.}

increases the weight of brown fat tissue and guanosine 5'diphosphate (GDP) binding ability, which is an index of thermogenesis, and significantly promotes urinary excretion of noradrenaline over a 24 -hour period ${ }^{15,16}$. Based on these findings, it is suggested that MCT activates the sympathetic nervous system and increases energy expenditure.

In view of the fact that MLCT, which we developed, contains only a small amount of MCFA, approximately $12 \%$, further study will be required to clarify the accelerative effect of MLCT intake on DIT and its suppressive effect on body fat accumulation. Kasai et al. investigated the suppressive effect of long-term MLCT intake on body fat accumulation $^{17)}$, and revealed that when $14 \mathrm{~g}$ of either LCT or MLCT was given to 82 volunteers, MLCT intake significantly reduced the amount of body fat, subcutaneous fat area, and visceral fat area. In our study, the liquid meal containing MLCT contained approximately $13.5 \mathrm{kcal}$ MCFA, and energy expenditure increased by approximately $13.6 \mathrm{kcal}$ as compared with LCT intake. That is, the MLCT-accelerated DIT suggests that almost all of the MCFA was consumed. However, Shinohara et al. assessed hepatic $\beta$-oxidizing system activity after intake of MLCT or MCT in rats, and found that the $\beta$-oxidizing system enzyme activity of MLCT was similar or superior to that of $\mathrm{MCT}^{18}$. This indicates that not only MCFA taken into the liver may be $\beta$-oxidized and metabolized, but also LCFA present in the surrounding area may also be simultaneously consumed. Based on the above results, MLCT intake is also considered to possibly accelerate DIT and suppress body fat accumulation by consuming LCFA in addition to MCFA.

This study has demonstrated that MLCT accelerates energy production and contributes to less accumulation of body fat. For future health promotion, widespread use of MLCT is expected to prevent obesity as well as metabolic syndrome.

\section{ACKNOWLEDGMENT}

We are deeply grateful to the staff of the Food Development Center of Nissin OilliO Group, Ltd. for cooperation in preparing the study food.

\section{References}

1. Matsuzawa, Y. The metabolic syndrome and adipocytokines. FEBS Lett. 580, 2917-2921 (2006).

2. Nagaretani, H.; Nakamura, T.; Funahashi, T.; Kotani, K.; Miyanaga, M.; Tokunaga, K.; Takahashi, M.; Nishizawa, H.; Kishida, K.; Kuriyama, H.; Hotta, K.; Yamashita, S.; Y. Matsuzawa. Visceral fat is a major contributor for multiple risk factor clustering in Japanese men with impaired glucose tolerance. Diabetes Care. 24, 2127-2133 (2001).

3. The Journal of the Japanese Society of Internal Medicine. 94, 794-809 (2005).

4. Summary of the results of the national health and nutrition survey conducted in 2004. Ministry of Health, Labour and Welfare of Japan.

5. Himms-Hagen, J. Thermogenesis in brown adipose tissue as an energy buffer. Implications for obesity. $N$. Engl. J. Med. 311, 1549-1558 (1984).

6. Suzuki, Y.; Nosaka, N.; Maki, H.; Kasai, M.; Aoyama, T.; Haruna, H.; Toda, T.; Okazaki, M.; Igarashi, O.; Kondo, $\mathrm{K}$. Effects of margarine containing medium-chain triglycerides on diet-induced thermogenesis. J. Oleo Sci. 54, 299-304 (2005).

7. St-Onge, M.P.; Jones, P.J. Greater rise in fat oxidation with medium-chain triglyceride consumption relative to long-chain triglyceride is associated with lower initial body weight and greater loss of subcutaneous adipose tissue. Int. J. Obes. 27, 1565-1571 (2003).

8. St-Onge, M.P.; Bourque, C.; Jones, P.J.; Ross, R.; Parsons, W.E. Medium- versus long-chain triglycerides for 27 days increases fat oxidation and energy expenditure without resulting in changes in body composition in overweight women. Int. J. Obes. 27, 95-102 (2003).

9. Nosaka, N.; Suzuki, Y.; Maki, H.; Haruna, H.; Ohara, A.; Kasai, M.; Tsuji, H.; Aoyama, T.; Okazaki, M.; Kondo, K. Effects of ingestion of margarine containing mediumchain triglycerides for 4weeks on blood parameters and postprandial thermogenesis. J. Oleo Sci. 52, 571-581 (2003).

10. Kasai, M.; Nosaka, N.; Maki, H.; Suzuki, Y.; Takeuchi, H.; Aoyama, T.; Ohara, A.; Harada, Y.; Okazaki, M.; Kondo, K. Comparison of diet-induced thermogenesis of foods containing medium- versus long-chain triacylglycerols. J. Nutr. Sci. Vitaminol. 48, 536-540 (2002).

11. Matsuo, T.; Matsuo, M.; Taguchi, N.; Takeuchi, H. The thermic effect is greater for structured medium- and long-chain triacylglycerols versus long-chain triacylglycerols in healthy young women. Metabolism. 50, 125-130 (2001).

12. Negishi, S.; Shirasawa, S.; Arai, Y.; Suzuki, J.; Mukataka, S. Activation of powdered lipase by cluster water and the use of lipase powders for commercial esterification of food oils. Enzyme Microb. Technol. 32, 66-70 (2003).

13. Weir, J.B. New methods for calculating metabolic rate with special reference to protein metabolism. J. Physiol. 109, 1-9 (1949).

14. Nosaka, N.; Maki, H.; Suzuki, Y.; Haruna, H.; Ohara, A.; Kasai, M.; Tsuji, H.; Aoyama, T.; Okazaki, M.; Igarashi, O.; Kondo, K. Effects of margarine containing mediumchain triacylglycerols on body fat reduction in humans. 
J. Ateroscler. Thromb. 10, 290-298 (2003).

15. Rothwell, N.J.; Stock, M.J. Stimulation of thermogenesis and brown fat activity in rats fed medium chain triglyceride. Metabolism. 36, 128-130 (1987).

16. Dulloo, A.G.; Fathi, M.; Mensi, N.; Girardier, L. Twenty-four-hour energy expenditure and urinary catecholamines of humans consuming low-to-moderate amounts of medium-chain triglycerides: a doseresponse study in a human respiratory chamber. Eur. J. Clin. Nutr. 50, 152-158 (1996).

17. Kasai, M.; Nosaka, N.; Maki, H.; Negishi, S.; Aoyama,
T.; Nakamura, M.; Suzuki, Y.; Tsuji, H.; Uto, H.; Okazaki, M.; Kondo, K. Effect of dietary medium- and longchain triacylglycerols (MLCT) on accumulation of body fat in healthy humans. Asia Pacific J. Clin. Nutr. 12, 151-160 (2003).

18. Shinohara, H.; Ogawa, A.; Kasai, M.; Aoyama, T. Effect of randomly interesterified triacylglycerols containing medium- and long-chain fatty acids on energy expenditure and hepatic fatty acid metabolism in rats. Biosci. Biotechnol. Biochem. 69, 1811-1818 (2005). 